\title{
Mucocutaneous Horn in Dog
}

\author{
Joyce Maira de Araújo', Stephanie Carrelo de Lima² \& Eric Schmidt Rondon²
}

\begin{abstract}
Background: Cutaneous horn is a circumscribed exophytic lesion composed of dense, compact keratin with hyperplastic epidermis, which is primarily orthokeratotic and may include foci of parakeratosis. The hyperkeratotic protuberance resembles a horn but lack bone. In humans, it is well-documented with a wide range of primary epidermal lesions identified. In dogs, the reports are rare and brief. The diagnosis is based on its appearance and excisional biopsy reveals the triggering lesion. The objective of the present work is to describe the clinical presentation, predictive benignancy finds, treatment and follow-up of a case of cutaneous horns that arose from anal mucocutaneous boundary in a dog.

Case: A 6-month-old male Pug presented two tumors in the anus noted in the early months of life. Complete blood count, serum chemistry profile, surgery, histopathological analysis and postoperative outcome were performed. Possible relapses were follow-up until 11 months after surgery. On physical examination, the dog was otherwise healthy. The two horn-like tumors were protruding from the mucocutaneous junction of the external anal sphincter in a sun-protected area. Both were higher than wide in base or, in other words, they had a high height-to-base ratio. During surgery, the masses were excised using an electronic scalpel and sent to histopathological analysis. The post-operative care consisted of cephalexin and meloxicam prescriptions. Also, it was recommended $0.9 \% \mathrm{NaCl}$ solution wound flushing followed by topical chlorhexidine digluconate solution (1\%) whenever dirt or defecation were noticed. The surgical wound healed after two weeks by second intention. Histopathology found well-delimited masses that covers the epidermis and dermis and displaces the cutaneous appendages towards the underlying musculature with an aggregate of lymphoplasmohistiocytic inflammatory infiltrate in the adjacent dermis. These finds and the presence of orthokeratotic hyperkeratosis and epidermis hyperplasia confirmed the clinical diagnosis of cutaneous horns. There were no signs of malignance. After surgery, no relapse occurred.

Discussion: To the best of the authors' knowledge, this is the first confirmed case of cutaneous horns in mucocutaneous junction. Furthermore, the tumors were found in a region with little exposure to sunlight which is unusual. The tumor's narrow bases and the absence of continuous and dense inflammatory infiltrate shown to be predictive of benignancy as occurs in human beings. During surgical planning, it was decided not to establish wide margins around the masses. The decision considered the morphological signs of benignancy of the tumors described for human beings as no surveys about prevalence of benignity or malignancy associated with cutaneous horns were found in dogs. In addition, a more extensive excision could promote anal sphincter dysfunction. There was no tumors recurrence suggesting that the primary underlying lesions have been healed and confirming that predictive benignancy morphological characteristics applied in human patients can be useful for small animals. Veterinary clinicians and surgeons must be in constant vigil of cutaneous horns uncommon presentations and report them to create a solid database that can be useful for prognosis and surgical planning. The morphological predictive factors can be applied to avoid unnecessary extensive surgical excisions that could lead to functional or cosmetic impairment.
\end{abstract}

Keywords: benignancy, cutaneous horns, predictive, malignancy, surgery, tumor. 


\section{INTRODUCTION}

The term cutaneous horn, or cornu cutaneum is a semiotic concept described in the late 16th century alluding to a yellowish, blackened or whitish, firm, conical and hyperkeratotic protrusion appearing an animal horn that ranges in size from a few millimeters to several centimeters [9]. Cutaneous horns are uncommon tumors in dogs [2] and the few existing reports are brief.

Diagnosis is made by appearance, course of injury, and biopsy [6] which must include tumor's hyperkeratotic base where primary benign or malignant lesions may be present [3].

In humans, cutaneous horns in sun-exposed skin areas [6] or with a wide base or a low heightto-base ratio were significantly more likely to show (pre)malignant base pathology [10]. In addition, inflammation around the cutaneous horn and dense inflammatory infiltrate at the base were associated with malignancy [5].

Treatment requires local excision with $1 \mathrm{~cm}$ margins to avoid recurrence [7].

To the best of the authors' knowledge, this is the first confirmed case of cutaneous horns in mucocutaneous junction. At this point, the objective of this report is to describe the clinical presentation, predictive benignancy finds, treatment and follow-up of a case of cutaneous horns that arose from anal mucocutaneous boundary in a dog.

\section{CASE}

A 6-month-old, male, Pug presented two horn-like tumors in the anus. The owner noted the masses in the first months of dog's life. There was no relevant data in past medical history.

Upon physical examination, vital signs were unchanged. Two horn-shaped tumors (Figure 1) arising from mucocutaneous junction of external anal sphincter were identified. The dorsal one was in 11 o'clock position and measured $1.7 \mathrm{~cm}$ in height and $0.7 \mathrm{~cm}$ in base $(1.7 \times 0.7 \mathrm{~cm}$; height-to-base ratio of 2.42$)$. The other one was in 7 o'clock position and had $1.0 \times 0.3$ $\mathrm{cm}$ (height-to-base ratio of 3.33).

Blood samples were collected for complete blood count (CBC), serum creatinine levels, alanine aminotransferase (ALT) and aspartate aminotransferase (AST) tests.
Both masses had consistent appearance with cutaneous horns and surgical removal was recommended.

After one week, the tumors were excised using an electronic scalpel and immediately sent for histopathological examination. Less than $1 \mathrm{~cm}$ of margins were left.

Cephalexin ${ }^{1} 30.0 \mathrm{mg} / \mathrm{kg}$ p.o. twice daily for 10 days and meloxicam ${ }^{2} 0.1 \mathrm{mg} / \mathrm{kg}$ p.o. daily for 3 days were prescribed. It was recommended Saline $(0.9 \%$ $\mathrm{NaCl}$ solution) wound flushing followed by topical chlorhexidine digluconate solution $(1 \%)$ whenever dirt or defecation were noticed.

Postoperative outcome was weekly for two weeks. Possible recurrences were observed following the removal of the tumors.

The dog was otherwise healthy, with normal diagnostic tests (CBC, serum creatinine, AST and ALT).

Macroscopically, the tumors were firm, conical, well circumscribed and after cut, grayed out.

Histopathology analysis showed that the formed masses comprised epidermis and dermis and displaced the cutaneous attachments towards the underlying musculature. The adjacent dermis had small aggregates of lymphoplasmohistiocytic inflammatory infiltrate (Figure 2). Each tumor was well delimited, not encapsulated, expansive and composed of marked epidermal hyperplasia, on which there were orthokeratotic hyperkeratosis and multifocal areas of parakeratotic hyperkeratosis (Figure 2). There were no finds of premalignant or malignant lesions.

Surgical wound healing was achieved after two weeks and no recurrences were observed until 11 months after surgery.

\section{DISCUSSION}

The term cutaneous horn refers to a morphological description [1] and so the diagnosis was based on tumor's appearance and confirmed by biopsy.

In the present case, the tumors were found in a region with little exposure to sunlight. There is no literature discussing cases of cutaneous horn on dogs with this presentation, but in persons this is an unusual location [6]. Both masses had narrow bases compared with their heights. In humans, these finds are predictive of benign primary lesions of cutaneous horn [10]. No similar data was found in veterinary literature. 
The excision of the tumors with cauterization of the margins without apposition with suture material (healing by secondary intention) was planned. Cryosurgery was indicated to removal of benign lesions associated with cutaneous horn [9]. Unfortunately, this procedure was unavailable at our hospital.

Given the location of the tumors, it was decided not to establish wide margins of $1.0 \mathrm{~cm}$ [7] to avoid the risk of anal sphincter dysfunction [8]. However, more extensive resections are also important for detecting the base lesion [3]. Thus, the performed surgery entailed two risks: the possibility to left premalignant or malignant lesions at the base and insufficient material for histopathological diagnosis. No surveys about prevalence of benignity or malignancy associated with cutaneous horns were found in dogs. In human beings, most of the lesions (61.1\%) are benign [10], but when occurring in sun exposed areas, $58.56 \%$ are premalignant or malignant [6]. Moreover, considering the morphological aspect of the masses, as discussed before, the first hypothesis was unlikely. In addition, in humans, inflammation around the cutaneous horn [5] and dense inflammatory infiltrate at the base are histopathological finds associated with malignancy $[4,5]$ which was not found in this clinical case as there was a space between the sparse lymphoplasmohistiocytic inflammatory infiltrate and the cutaneous horns (Figure 2).

Finally, there was no tumors recurrence suggesting that the primary underlying lesions have been healed

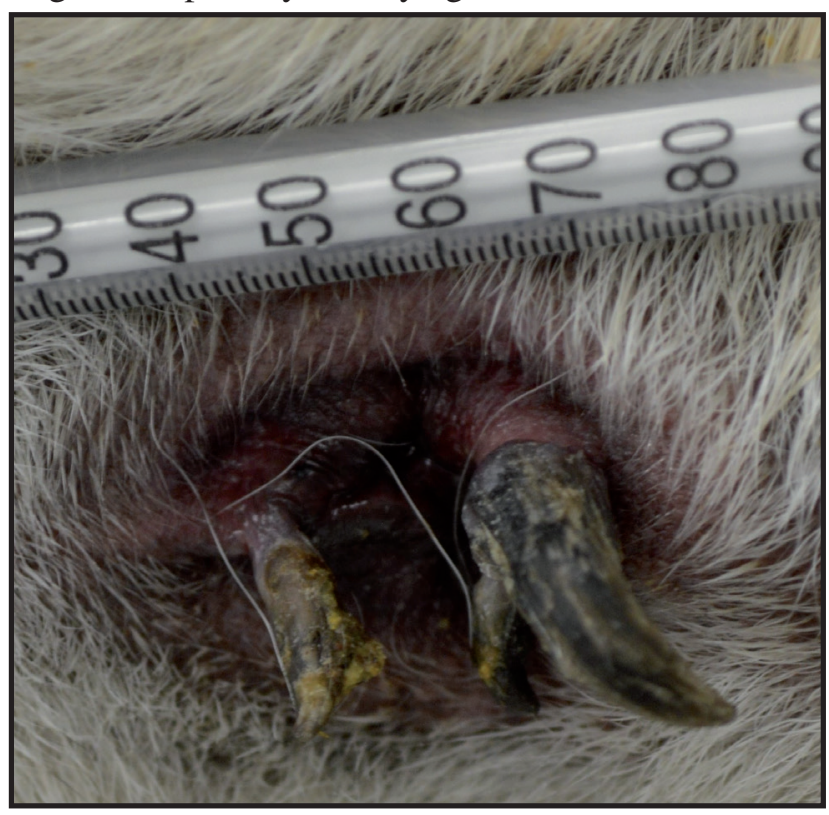

Figure 1. Clinical photographs of the perianal area with a 100 UI syringe used as a size parameter. A 6-month-old, male, Pug in right lateral recumbency. Note there are two tumors resemble horns (cutaneous horns) arising from mucocutaneous junction of external anal sphincter. Both were higher than wide in base. by the removal or complete cauterization. In a study with human patients, the absence of relapse three months after surgery indicated well succeeded treatment [7].

Further studies are required to explore cutaneous horn's clinical and histopathological finds in order to optimize prognosis and surgical planning. Given the background, morphological predictive factors of benignancy can be applied to avoid unnecessary extensive surgical excisions that could lead to functional or cosmetic impairment.

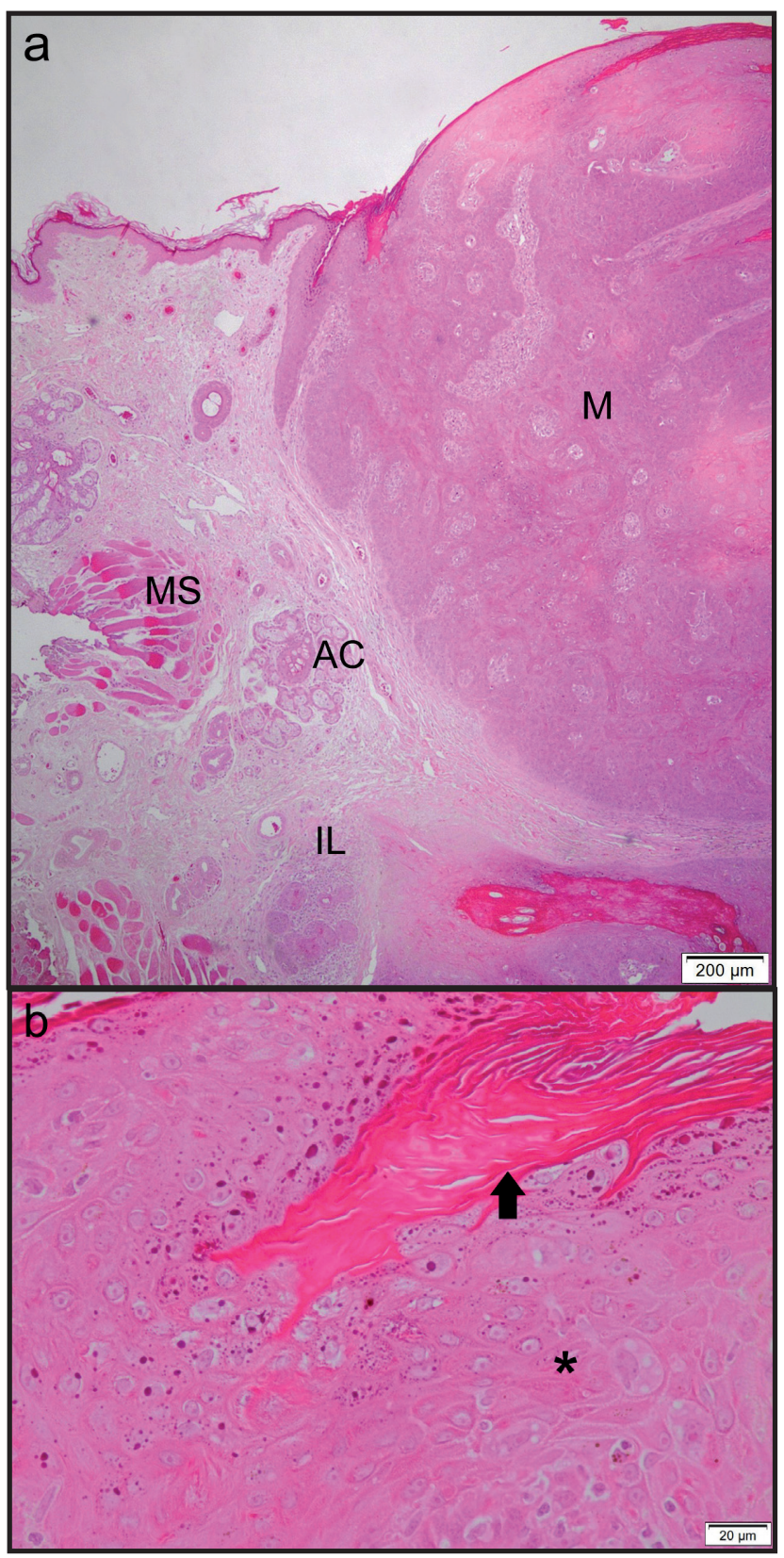

Figure 2. Photomicrographs of skin samples plus cutaneous horns. A 6-month-old, male, Pug. a- Well delimited mass (M) that covers the epidermis and dermis and displaces the cutaneous appendages (AC) towards the underlying musculature (MS). An aggregate of lymphoplasmohistiocytic inflammatory infiltrate in the adjacent dermis (IL) [H\&E; x4, Bar= $200 \mu \mathrm{m}] . \mathrm{b}$ - There is orthokeratotic hyperkeratosis (arrow) and epidermis hyperplasia $\left(^{*}\right)[\mathrm{H} \& \mathrm{E} ; \mathrm{x} 40, \mathrm{Bar}=20 \mu \mathrm{m}]$. 


\section{MANUFACTURERS}

${ }^{1}$ ABL. Antibióticos do Brasil. São Paulo, SP, Brazil. ${ }^{2}$ Eurofarma Laboratórios S.A. Ribeirão Preto, SP, Brazil.
Declaration of interest. The authors report no conflicts of interest. The authors alone are responsible for the contents and writing of the paper.

\section{REFERENCES}

1 Falk E., Lange C.E., Jennings S. \& Ferrer L. 2017. Two cutaneous horns associated with canine papillomavirus type 1 infection in a Pit Bull dog. Veterinary Dermatology. 28(4): 420-421.

2 Goldschmidt M.H. \& Goldschmidt K.H. 2016. Epithelial and Melanocytic Tumors of the Skin. In: Meuten D.J. (Ed). Tumors in Domestic Animals. 5th edn. Ames: Wiley-Blackwell, pp.88-141.

3 Hermida Pérez J.A. \& Bermejo Hernández A. 2013. Cutaneous horn, actinic keratosis and squamous cell carcinoma. Presentation of a clinical case. SEMERGEN - Medicina de Familia. 39(2): 113-116.

4 Jakubowska K., Kisielewski W., Kańczuga-Koda L., Koda M. \& Famulski W. 2017. Diagnostic value of inflammatory cell infiltrates, tumor stroma percentage and disease-free survival in patients with colorectal cancer. Oncology Letters. 14(3): 3869-3877.

5 Kastanioudakis I., Skevas A., Assimakopoulos D. \& Daneilidis B. 1998. Cutaneous horn of the auricle. Otolaryngology - Head And Neck Surgery. 118(5): 735.

6 Mantese S.A.O., Diogo P.M., Rocha A., Berbert A.L.C.V., Ferreira A.K.M. \& Ferreira T.C. 2010. Cutaneous horn: a retrospective histopathological study of 222 cases. Anais Brasileiros de Dermatologia. 85(2): 157-163.

7 Mir M.A., Mahmud A.A., Mohd Y.M.C., Rehman S., Imran A.M.C. \& Lalit M.B.M.C. 2016. Cutaneous Horn: A Devil Not Only in Appearance. Plastic Surgery Case Studies. 2(3): 51-52.

8 Rodaski S., Guérios S.D., Koppe A.B., Sincero P.C., Tranquilin M.V., Perroni M.A. \& Nardi A.B. 2000. Experimental myoplasty of the external anal sphincter with autologous fascia Lata in dogs. Archives of Veterinary Science. 5: 49-54.

9 Sabando Carranza J.A. \& Calvo Carrasco D. 2011. Cuerno cutáneo. Formación Médica Continuada en Atención Primaria. 18(10): 656-657.

10 Yu R.C.H., Pryce D.W., Macfarlane A.W. \& Stewart T.W. 1991. A histopathological study of 643 cutaneous horns. British Journal of Dermatology. 124(5): 449-452. 